\title{
The dynamic logic of stating and asking: a study of inquisitive dynamic modalities
}

\author{
Ivano Ciardelli \\ ILLC, University of Amsterdam
}

\begin{abstract}
Inquisitive dynamic epistemic logic (IDEL) extends standard public announcement logic incorporating ideas from inquisitive semantics. In IDEL, the standard public announcement action can be extended to a more general public utterance action, which may involve a statement or a question. While uttering a statement has the effect of a standard announcement, uttering a question typically leads to new issues being raised. In this paper, we investigate the logic of this general public utterance action. We find striking commonalities, and some differences, with standard public announcement logic. We show that dynamic modalities admit a set of reduction axioms, which allow us to turn any formula of IDEL into an equivalent formula of static inquisitive epistemic logic. This leads us to establish several complete axiomatizations of IDEL, corresponding to known axiomatizations of public announcement logic.
\end{abstract}

\section{Introduction}

Dynamic epistemic logics $[11,2]$ allow us to reason about how an epistemic scenario evolves when certain actions are performed. The simplest kind of action considered in these logics is the public announcement of a formula $[16,12,1,10]$. When $\varphi$ is publicly announced, all agents learn that $\varphi$ was true at the time of the announcement, and that this is now common knowledge among the group. The action of announcing a formula $\varphi$ is associated with a dynamic modality $[\varphi]$, which can be used to relativize a formula to the situation that results from an announcement of $\varphi$. The resulting dynamic logic, PAL, admits reduction axioms: these are a set of equivalences that allow us to recursively eliminate the dynamic modality, transforming each formula of PAL into a corresponding formula of static epistemic logic. A complete axiomatization of PAL is obtained combining the reduction axioms with a complete axiomatization of epistemic logic.

In recent years, PAL and other dynamic epistemic logics have been fruitfully employed to analyze processes of information exchange $[11,2]$. However, a typical information exchange process is not a mere sequence of announcements. Rather, it can be seen as a process in which certain issues are raised, addressed, and possibly resolved. Typically, issues are raised by asking questions, and resolved by making statements. Starting from this idea, Ciardelli and Roelofsen [9] introduced inquisitive epistemic logic (IEL). This framework describes not just the information that agents have, but also the information that they would like to obtain, i.e., the issues they are interested in. Accordingly, the logical language of 
IEL can talk not just about the agent's information, but also about their issues. This is achieved by enriching classical logic with questions (drawing on work in inquisitive logic $[3,8,7])$ and by letting modal operators embed questions as well as statements. An important feature of IEL is that its modalities, while more expressive than standard Kripke modalities, are logically very well-behaved. A complete axiomatization of the resulting modal logic was established in [4].

Ciardelli and Roelofsen [9] also generalized the standard account of public announcements to the inquisitive setting. In the resulting inquisitive dynamic epistemic logic (IDEL), agents can not only provide new information by publicly making a statement, but also raise new issues by publicly asking a question. Thus, IDEL provides the tools for a basic modeling of communication as a process in which agents interact by raising and resolving issues. As in PAL, the action of uttering a formula $\varphi$ is associated with a dynamic modality $[\varphi]$, whose effect is to relativize a formula to the situation that results from the utterance of $\varphi$.

In this paper we investigate and axiomatize the resulting dynamic logic. We will show that the key features of PAL are preserved in IDEL, although there are also a few interesting differences. Like in PAL, it is possible to identify a set of logical equivalences by means of which any IDEL-formula can be turned into an equivalent formula of static IEL. In combination with the completeness result for IEL given in [4], this yields a complete axiomatization of IDEL. This provides a proof system that can be used to reason about how a communication scenario evolves when questions are publicly asked, or statements are publicly asserted.

The paper is structured as follows: Sections 2 and 3 provide an introduction to IDEL. Sections 4 and 5 present results on the logic of dynamic modalities, which are used in Section 6 to obtain a complete axiomatization. Section 7 concludes.

\section{Inquisitive epistemic logic}

In this section, we provide a concise introduction to the framework of inquisitive epistemic logic, following [5]. For discussion and proofs, we refer to [9, 5].

The main ingredients for the semantics are the notions of information states and issues. The former is standard, while the latter stems from work on inquisitive semantics [6]. An information state is modeled by identifying it with the set of worlds compatible with it. Similarly, an issue is modeled by identifying it with the set of information states where it is resolved.

Definition 1 (States and issues). If $\mathcal{W}$ is a set of possible worlds, then:

- an information state is a subset $s \subseteq \mathcal{W}$;

- an issue is a non-empty set I of information states that is downward closed: if $s \in I$ and $t \subseteq s$, then $t \in I$. The set of all issues is denoted by $\mathcal{I}$.

The downward closure condition captures the fact that if an issue $I$ is resolved in $s$, and if $t$ contains all the information that $s$ contains, then $I$ is resolved in $t$. The maximal elements in an issue $I$ will be called the alternatives. Notice that $I$ can only be truthfully resolved at a world $w$ if some resolving state $s \in I$ contains $w$, i.e., if $w \in \bigcup I$. We will say that $I$ is an issue over the state $\bigcup I$. 
Standard epistemic models describe situations which are determined by the truth value of certain primitive facts and by the knowledge of certain agents. Accordingly, a possible world $w$ is fully described by (i) a propositional valuation $V(w)$, specifying which atomic sentences are true at $w$ and (ii) for every agent $a$, an information state $\sigma_{a}(w)$, representing the knowledge of $a$ in $w$. In inquisitive epistemic logic, what matters is not only the knowledge that agents have, but also the issues that they entertain. Thus, the description of a possible world $w$ includes, for every agent $a$, an issue $\Sigma_{a}(w)$ over $\sigma_{a}(w)$, called the inquisitive state of $a$ in $w: s$ belongs to $\Sigma_{a}(w)$ iff all the issues entertained by $a$ in $w$ are resolved in $s$. Intuitively, we think of $a$ as aiming to reach one of the states $s \in \Sigma_{a}(w)$. Since $\Sigma_{a}(w)$ is required to be an issue over $\sigma_{a}(w)$, we have $\sigma_{a}(w)=\bigcup \Sigma_{a}(w)$. Hence, the map $\Sigma_{a}$ by itself encodes both $a$ 's knowledge and $a$ 's issues, and we do not need $\sigma_{a}$ as a separate component in the model. As in standard epistemic logic, the maps $\Sigma_{a}$ may be constrained by specific requirements. Following [9], we build on the strongest version of epistemic logic, which requires factivity and introspection, where the latter now concerns both information and issues.

Definition 2 (Inquisitive epistemic models). An inquisitive epistemic model for a set $\mathcal{P}$ of atoms and a set $\mathcal{A}$ of agents is a triple $M=\left\langle\mathcal{W}, \Sigma_{\mathcal{A}}, V\right\rangle$ where $\mathcal{W}$ is a set (the possible worlds of the model), $V: \mathcal{W} \rightarrow \wp(\mathcal{P})$ is a valuation map, and $\Sigma_{\mathcal{A}}=\left\{\Sigma_{a} \mid a \in \mathcal{A}\right\}$ is a set of inquisitive state maps $\Sigma_{a}: \mathcal{W} \rightarrow \mathcal{I}$, each of which assigns to every world $w$ an issue $\Sigma_{a}(w)$, in accordance with

- Factivity: for any $w \in \mathcal{W}, w \in \sigma_{a}(w)$

- Introspection: for any $w, v \in \mathcal{W}$, if $v \in \sigma_{a}(w)$, then $\Sigma_{a}(v)=\Sigma_{a}(w)$

where $\sigma_{a}(w):=\bigcup \Sigma_{a}(w)$ represents the epistemic state of agent a in $w$.

A useful way to draw the state map of an agent is illustrated in the figure below. At a world $w$, the epistemic state of the agent consists of those worlds included in the same dashed area as $w$; the solid blocks inside this area are the alternatives for the issue entertained at $w$ - the maximal states in which the issue is resolved.

Thus, at $w_{1}$ and $w_{2}$, the agent's epistemic state is $\left\{w_{1}, w_{2}\right\}$; the alternatives for the issue entertained by the agent are $\left\{w_{1}\right\}$ and $\left\{w_{2}\right\}$, which means that the agent is interested in whether $w_{1}$ or $w_{2}$ is actual. At worlds $w_{3}$ and $w_{4}$, the agent's epistemic state is $\left\{w_{3}, w_{4}\right\}$, and the unique alternative for the issue entertained by the agent is $\left\{w_{3}, w_{4}\right\}$, which means that the agent is not interested in acquiring any more specific information.

The language of IEL, $\mathcal{L}^{\mathrm{IEL}}$, is given by the following syntax,

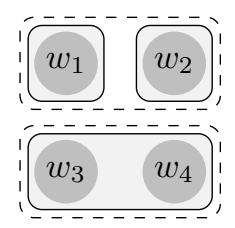
where $p \in \mathcal{P}$ is an atomic sentence and $a \in \mathcal{A}$ an agent label: ${ }^{1}$

\footnotetext{
${ }^{1}$ Most previous presentations of IEL $([9,4])$ use a dichotomous language, in which formulas are divided into two syntactic categories: declaratives and interrogatives. The application of connectives is then subject to syntactic restrictions. Here we follow [5] in using a more general, non-dichotomous language; connectives apply without any restrictions, which leads to a more elegant logic. This difference is not an essential one; the results obtained here can be adapted to the dichotomous setting.
} 


$$
\varphi::=p|\perp| \varphi \wedge \varphi|\varphi \rightarrow \varphi| \varphi \mathbb{V} \varphi\left|K_{a} \varphi\right| E_{a} \varphi
$$

The non-standard items in this language are the connective $\mathbb{V}$, called inquisitive disjunction and the modality $E_{a}$, which we read as entertain. As we shall see, former allows us to form questions, while the latter allows us to talk about the questions that an agent is interested in. We use the following abbreviations:

$$
\begin{array}{ll}
-\neg \varphi:=\varphi \rightarrow \perp & -\varphi \leftrightarrow \psi:=(\varphi \rightarrow \psi) \wedge(\psi \rightarrow \varphi) \\
-\varphi \vee \psi:=\neg(\neg \varphi \wedge \neg \psi) & -? \varphi:=\varphi \mathbb{\vee} \neg \varphi
\end{array}
$$

Usually, modal formulas are interpreted in terms of truth-conditions with respect to a possible world. However, the language of IEL comprises not only statements, but also questions, which are not naturally analyzed in terms of truth-conditions. Instead, following inquisitive semantics $[8,13]$, the semantics of IEL is given by a relation of support between formulas and information states.

Definition 3 (Support). Let $M$ be a model and $s$ an information state in $M$.

1. $M, s \models p \Longleftrightarrow p \in V(w)$ for all worlds $w \in s$

2. $M, s=\perp \Longleftrightarrow s=\emptyset$

3. $M, s \models \varphi \wedge \psi \Longleftrightarrow M, s \models \varphi$ and $M, s \models \psi$

4. $M, s \models \varphi \mathbb{V} \Longleftrightarrow M, s \models \varphi$ or $M, s \models \psi$

5. $M, s \models \varphi \rightarrow \psi \Longleftrightarrow$ for every $t \subseteq s, M, t=\varphi$ implies $M, t \models \psi$

6. $M, s \models K_{a} \varphi \Longleftrightarrow$ for every $w \in s, M, \sigma_{a}(w) \models \varphi$

7. $M, s \models E_{a} \varphi \Longleftrightarrow$ for every $w \in s$ and every $t \in \Sigma_{a}(w), M, t \models \varphi$

The support-set of $\varphi$ in $M$ is the set $[\varphi]_{M}:=\{s \subseteq \mathcal{W}|M, s|=\varphi\}$.

A key feature of the support relation is that it is persistent: that is, more formulas become supported as information grows: if $M, s \models \varphi$ and $t \subseteq s$, then $M, t \models \varphi$. As a limit case, $\emptyset$ supports all formulas: we refer to $\emptyset$ as the inconsistent state.

Although support at a state is the primitive semantic notion in IEL, truth at a world can be defined as support at the corresponding singleton.

Definition 4 (Truth).

We say that $\varphi$ is true at a world $w$, notation $M, w \models \varphi$, in case $M,\{w\} \models \varphi$. The truth-set of $\varphi$ in $M$ is the set $|\varphi|_{M}=\{w \in \mathcal{W}|M, w|=\varphi\}$.

Spelling out the support clauses for a singleton state, one can check that standard formulas receive the usual truth-conditions. In addition, the truth-conditions for modal formulas are as follows:

$-M, w=K_{a} \varphi \Longleftrightarrow M, \sigma_{a}(w)=\varphi$

$-M, w=E_{a} \varphi \Longleftrightarrow$ for every $t \in \Sigma_{a}(w), M, t=\varphi$

Notice that the truth-conditions of a modal formula $K_{a} \varphi$ or $E_{a} \varphi$ depend crucially on the support conditions of $\varphi$, and not just on its truth-conditions.

A formula is said to be truth-conditional in case support at an information state $s$ boils down to truth at each world $w \in s$. 
Definition 5 (Truth-conditionality). A formula $\varphi$ is truth-conditional if for all models $M$ and information states $s: M, s \models \varphi \Longleftrightarrow M, w \models \varphi$ for all $w \in s$.

We refer to truth-conditional formulas as statements and to non-truth-conditional formulas as questions [5]. Intuitively, we read $s \models \varphi$ as " $\varphi$ is known to be true in $s$ " if $\varphi$ is a statement, and as " $\mu$ is settled in $s$ " if $\varphi$ is a question.

It is often possible to tell from the form of $\varphi$ that it is truth-conditional. We define a set $\mathcal{L}_{!}^{\mathrm{ILL}}$ of declaratives as follows, where $\varphi \in \mathcal{L}^{\mathrm{IEL}}$ is any formula:

$$
\alpha::=p|\perp| K_{a} \varphi\left|E_{a} \varphi\right| \alpha \wedge \alpha \mid \varphi \rightarrow \alpha
$$

In other words, $\varphi$ is a declarative if the only occurrences of $\mathbb{V}$ in $\varphi$, if any, are within the scope of a modality or in a conditional antecedent. Then we have:

Fact 1 Any $\alpha \in \mathcal{L}_{!}^{\text {IEL }}$ is truth-conditional.

In particular, $\mathbb{V}$-free formulas are truth-conditional; also, modal formulas are always truth-conditional, even when the argument of the modality is a question.

With this background in mind, let us turn to an illustration of the system. First consider a standard propositional formula $\alpha$. It follows from Fact 1 that the meaning of $\alpha$ is completely determined by its truth-conditions, which are the standard ones. So, the meaning of $\alpha$ is essentially the same as in classical logic.

For an example of a question, consider the formula $? p:=p \Downarrow \neg p$. We have: $s \models ? p \Longleftrightarrow s \models p \llbracket \neg p \Longleftrightarrow s \mid=p$ or $s \models \neg p \Longleftrightarrow s \subseteq|p|_{M}$ or $s \subseteq|\neg p|_{M}$. Thus, a state supports ? $p$ if it implies either that $p$ is true, or that $p$ is false. Notice that, in any model containing both $p$-worlds and $\neg p$-worlds, the question $? p$ has two alternatives (two maximal supporting states) as shown in Figure 1(d).

Now consider the modalities. Since Fact 1 ensures that modal formulas are always truth-conditional - even when they embed a question - in order to understand the semantics of $K_{a} \varphi$ and $E_{a} \varphi$ we need only look at truth-conditions. First, consider the case that $\varphi$ is a statement $\alpha$. In this case, the two modalities coincide with each other and with the standard box modality of epistemic logic:

$$
M, w=K_{a} \alpha \Longleftrightarrow M, w \models E_{a} \alpha \Longleftrightarrow M, v \models \alpha \text { for all } v \in \sigma(w)
$$

Now consider the case in which $\varphi$ is a question $\mu$. Recall that, for a question, being supported amounts to being settled. Thus, the clauses read as follows:

- $K_{a} \mu$ is true at $w$ if $\mu$ is settled in the epistemic state $\sigma_{a}(w)$ of agent $a$ at $w$;

- $E_{a} \mu$ is true at $w$ if $\mu$ is settled in any information state where $a$ 's issues at $w$ are settled; that is, if settling the agent's issues implies settling $\mu$.

E.g., the formula $K_{a} ? p$ is true when the question ? $p$ is settled in $a$ 's epistemic state -i.e., when a knows whether $p$. By means of the modalities $K_{a}$ and $E_{a}$, a wondering modality is defined: $W_{a} \varphi:=\neg K_{a} \varphi \wedge E_{a} \varphi$. The idea is that $a$ wonders about a question if her current epistemic state does not settle the question, but she wants to reach a state that does settle the question.

As a concrete illustration, consider the model $M$ in Figure 1. At any world $w$ in this model, agent $a$ knows whether $p\left(M, w \models K_{a}\right.$ ? $\left.p\right)$; agent $b$ doesn't know whether $p$, but wonders about it $\left(M, w \models W_{b}\right.$ ? $\left.p\right)$; agent $c$ doesn't know, and does not care $\left(M, w \models \neg K_{c} ? p \wedge \neg W_{c} ? p\right)$. 


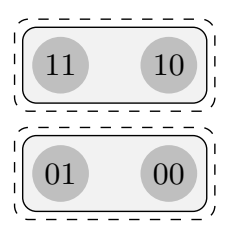

(a) Agent $a$

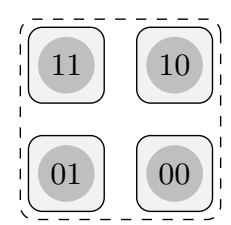

(b) Agent $b$

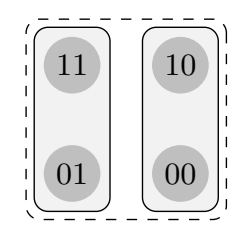

(c) Agent $c$
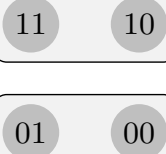

(d) $? p$

Fig. 1. A model for three agents $a, b, c$, and the alternatives for ? $p$.

\section{Uttering statements and questions}

In this section we review how the standard account of public announcements in epistemic logic can be lifted to a general account of public utterance in IEL [9]. ${ }^{2}$ Let us start out by specifying how an model changes as a result of the public utterance of a sentence $\varphi$. This works much like in PAL: when $\varphi$ is uttered, the worlds in which $\varphi$ is false are dropped from the model, and the state of each agent at a world is restricted by intersecting it with the support set $[\varphi]_{M}$.

Definition 6 (Updating a model). The update of a model $M=\left\langle W, \Sigma_{\mathcal{A}}, V\right\rangle$ with $\varphi \in \mathcal{L}^{I D E L}$ is the model $M^{\varphi}=\left\langle W^{\varphi}, \Sigma_{\mathcal{A}}^{\varphi}, V^{\varphi}\right\rangle$, where $W^{\varphi}=W \cap|\varphi|_{M}$, $V^{\varphi}=V \uparrow_{|\varphi|_{M}}$, and $\Sigma_{a}^{\varphi}(w)=\Sigma_{a}(w) \cap[\varphi]_{M}$.

The following fact says that the epistemic state $\sigma_{a}^{\varphi}(w):=\bigcup \Sigma_{a}^{\varphi}(w)$ of an agent at a world in the updated model is obtained just like in PAL, by restricting the original epistemic state $\sigma_{a}(w)$ to the set $|\varphi|_{M}$ of worlds where $\varphi$ is true.

Fact $2 \sigma_{a}^{\varphi}(w)=\sigma_{a}(w) \cap|\varphi|_{M}$

The language $\mathcal{L}^{\text {IDEL }}$ of IDEL extends the static language of IEL by allowing us to conditionalize a formula to the utterance of another formula. Formally, we have:

$$
\varphi::=p|\perp| \varphi \wedge \varphi|\varphi \rightarrow \varphi| \varphi \mathbb{V} \varphi\left|K_{a} \varphi\right| E_{a} \varphi \mid[\varphi] \varphi
$$

When talking of a formula $[\varphi] \psi$, we will refer to $\varphi$ as the label of the dynamic modality $[\varphi]$, and to $\psi$ as the argument. Semantically, assessing a sentence of the form $[\varphi] \psi$ at a model-state pair $\langle M, s\rangle$ requires assessing $\psi$ at the pair $\left\langle M^{\varphi}, s \cap|\varphi|_{M}\right\rangle$ of the updated model $M^{\varphi}$ and the restriction of $s$ to this model. That is, the semantics of IDEL extends Definition 3 with the following clause:

$$
M, s=[\varphi] \psi \stackrel{\text { def }}{\Longleftrightarrow} M^{\varphi}, s \cap|\varphi|_{M} \models \psi
$$

\footnotetext{
${ }^{2}$ We use the neutral term utterance rather than announcement (used in [9]) because the latter suggests an informational interpretation. E.g., in IDEL, the utterance of a question such as ? $p$ has the effect of raising the issue whether $p$. This should not be confused with the action of announcing whether $p$, i.e., announcing the true answer to the question ? $p$, which is a more standard action of providing information.
} 


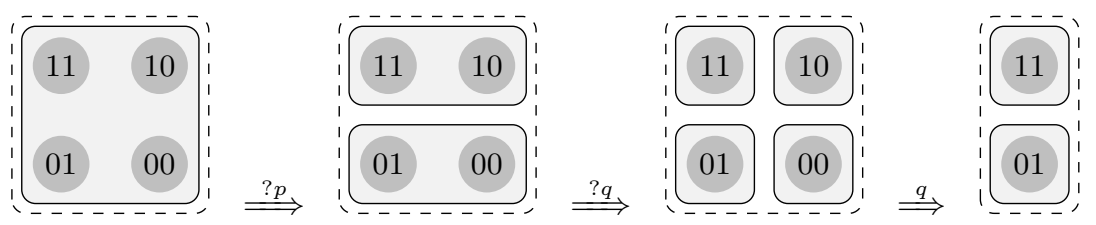

Fig. 2. The effects of a sequence of utterances on the state of an agent.

Specializing this clause to singleton information states, we recover the truthconditions that are familiar from PAL:

$$
M, w \models[\varphi] \psi \Longleftrightarrow M, w \models \varphi \text { implies } M^{\varphi}, w \models \psi
$$

To familiarize with the effects of the public utterance action, consider first the utterance of a truth-conditional formula $\alpha$. If $I$ is an issue and $s$ an information state, the restriction of $I$ to $s$ is the issue $I\lceil s=\{t \cap s \mid t \in I\}$. Then, we have:

Fact 3 If $\alpha \in \mathcal{L}^{I D E L}$ is truth-conditional, then $\Sigma_{a}^{\alpha}(w)=\Sigma_{a}(w)\left\lceil|\alpha|_{M}\right.$

Thus, there is nothing more to the utterance of a statement $\alpha$ than there is in standard PAL: as a consequence of the utterance, worlds where $\alpha$ was false are removed from the model, and the agents' states are restricted accordingly.

Now consider the utterance of a question, say a basic polar question ?p. Since $? p$ is true at all worlds, no world is removed from the model in the update. Moreover, by Fact 2 we have $\sigma_{a}^{? p}(w)=\sigma_{a}(w) \cap|? p|_{M}=\sigma_{a}(w)$, i.e., no knowledge is gained from an utterance of ? $p$. However, the update changes the inquisitive state of an agent $a$, from $\Sigma_{a}(w)$ to $\Sigma_{a}^{? p}(w)=\Sigma_{a}(w) \cap[? p]_{M}=\{s \mid s \in$ $\Sigma_{a}(w)$ and $\left.M, s \models ? p\right\}$. This means that $a$ 's issues after the utterance of ?p become more demanding: to settle them, a state must settle $a$ 's previous issues, and in addition the question ?p. In other words, as a result of the utterance, the agent will come to entertain the question ? $p$. This illustrates how uttering a question typically results in new issues being raised. ${ }^{3}$ The effect of a sequence of utterances on the state of an agent is shown in Fig. 2.

Let us now look at the significance of a dynamic formula of the form $[\varphi] \psi$. First, consider the special case in which $\psi$ is a statement, i.e., truth-conditional. Then, the following fact ensures that the whole formula $[\varphi] \psi$ is also a statement.

Fact 4 If $\alpha$ is truth-conditional, then so is $[\varphi] \alpha$ for any $\varphi$.

Thus, the semantics of $[\varphi] \alpha$ is fully captured by the truth-conditions of $[\varphi] \alpha$. These truth-conditions are standard: $[\varphi] \alpha$ is true if $\varphi$ is false, or $\varphi$ is true and $\alpha$

\footnotetext{
${ }^{3}$ It is not always the case that asking a question provides no information. In the case of a question having a non-trivial presupposition, like $p \llbracket q$, asking it has the effect of conveying the information corresponding to the presupposition, $p \vee q$. An alternative account would regard this information as something that has to be established before the question can be appropriately asked (cf. $[15,14])$. A modification of the present account of the public utterance action in this sense is proposed in $\S 3.2 .4-3.2 .5$ of [9].
} 
is true in the model resulting from the update with $\varphi$. Thus, as in PAL, we can read $[\varphi] \alpha$ as stating that $\alpha$ would be the case after an announcement of $\varphi{ }^{4}$

Now consider the case of a formula $[\varphi] \mu$, where $\mu$ is a question. In this case, $[\varphi] \mu$ can be thought of as a "dynamic conditional question", which asks to resolve $\mu$ under the assumption not just that $\varphi$ is true, but that $\varphi$ were uttered. As an example, consider the formula $[p]$ ? $K_{a} q$.

$$
\begin{aligned}
M, s \models[p] ? K_{a} q \Longleftrightarrow & \text { for all } w \in s \cap|p|_{M}, \sigma_{a}(w) \cap|p|_{M} \subseteq|q|_{M} \text { or } \\
& \text { for all } w \in s \cap|p|_{M}, \sigma_{a}(w) \cap|p|_{M} \nsubseteq|q|_{M}
\end{aligned}
$$

Thus, $[p] ? K_{a} q$ is settled if either (i) it is settled that, if $p$ is true and $a$ were to learn that $p$, she would know that $q$, or (ii) it is settled that, if $p$ is true and $a$ were to learn that $p$, she would still not know that $q$. Thus, $[p]$ ? $K_{a} q$ captures the question: "if $p$ were publicly uttered, would $a$ know that $q$ ?". And indeed, we have $[p] ? K_{a} q \equiv[p] K_{a} q \rrbracket[p] \neg K_{a} q$, which shows that $[p] ? K_{a} q$ can be settled either by establishing that $[p] K_{a} q$, or by establishing that $[p] \neg K_{a} q$.

\section{Normal form}

In this section, we begin our study of the logic of IDEL by establishing a normal form result which will turn out useful in further investigating the logic. The first step is to generalize to IDEL the notion of declaratives given above for IEL. The set $\mathcal{L}_{!}^{\text {IDEL }}$ of declarative formulas of IDEL is defined as follows, where $\varphi \in \mathcal{L}^{\text {IDEL}}$ :

$$
\alpha::=p|\perp| K_{a} \varphi\left|E_{a} \varphi\right|[\varphi] \alpha|\alpha \wedge \alpha| \varphi \rightarrow \alpha
$$

Thus, a declarative is a formula in which any occurrence of $\mathbb{V}$ is either (i) within the scope of a static modality $K_{a}$ or $E_{a}$ or (ii) within the label of a dynamic modality or (iii) within a conditional antecedent. The following fact can then be shown by means of a straightforward inductive proof.

Proposition 1. Any declarative formula $\alpha \in \mathcal{L}_{!}^{\text {IDEL }}$ is truth-conditional.

Next, we associate with any formula $\varphi \in \mathcal{L}^{\mathrm{IDEL}}$ a set $\mathcal{R}(\varphi) \subseteq \mathcal{L}_{!}^{\mathrm{IDEL}}$ of declaratives such that $\varphi$ is equivalent with the inquisitive disjunction of the $\alpha \in \mathcal{R}(\varphi)$. This shows that any formula of IDEL is equivalent to an inquisitive disjunction of truth-conditional formulas. This fact will be useful in the next two sections. The inductive proof of the normal form result is given in Appendix A.1.

\section{Definition 7 (Resolutions).}

$-\mathcal{R}(\alpha)=\{\alpha\}$ if $\alpha$ is of the form $p, \perp, K_{a} \varphi$ or $E_{a} \varphi$

$-\mathcal{R}(\varphi \wedge \psi)=\{\alpha \wedge \beta \mid \alpha \in \mathcal{R}(\varphi)$ and $\beta \in \mathcal{R}(\psi)\}$

$-\mathcal{R}(\varphi \mathbb{\psi} \psi)=\mathcal{R}(\varphi) \cup \mathcal{R}(\psi)$

$-\mathcal{R}(\varphi \rightarrow \psi)=\left\{\bigwedge_{\alpha \in \mathcal{R}(\varphi)} \alpha \rightarrow f(\alpha) \mid f: \mathcal{R}(\varphi) \rightarrow \mathcal{R}(\psi)\right\}$

$-\mathcal{R}([\varphi] \psi)=\{[\varphi] \alpha \mid \alpha \in \mathcal{R}(\psi)\}$

\section{Theorem 1 (Normal form for IDEL).}

Let $\varphi \in \mathcal{L}^{I D E L}$ and let $\mathcal{R}(\varphi)=\left\{\alpha_{1}, \ldots, \alpha_{n}\right\}$. Then $\varphi \equiv \alpha_{1} \mathbb{V} \ldots \mathbb{V} \alpha_{n}$.

\footnotetext{
${ }^{4}$ In particular, formulas from the language of PAL have the standard truth-conditions.
} 


\section{Reduction}

In this section, we will show that the presence of dynamic modalities does not make IDEL more expressive than its static fragment, IEL. As in PAL, any occurrence of a dynamic modality can be paraphrased away, inductively on the structure of the argument. This is easy if the argument is an atom or $\perp$.

Proposition 2. $[\varphi] p \equiv \varphi \rightarrow p$ and $[\varphi] \perp \equiv \neg \varphi$

Proof. Notice that both $[\varphi] p$ and $\varphi \rightarrow p$ are declaratives, and thus truthconditional by Proposition 1. Thus, to establish the equivalence we just have to show that these formulas have the same truth-conditions. We have:

$$
\begin{aligned}
M, w \mid=[\varphi] p & \Longleftrightarrow M, w \models \varphi \text { implies } M^{\varphi}, w \models p \\
& \Longleftrightarrow M, w \models \varphi \text { implies } M, w \models p \quad \Longleftrightarrow M, w \models \varphi \rightarrow p
\end{aligned}
$$

The equivalence $[\varphi] \perp \equiv \neg \varphi$ is established by an analogous argument.

As in PAL, dynamic modalities distribute smoothly over the connectives, which now also include inquisitive disjunction. The distribution over $\wedge$ and $\mathbb{V}$ is immediate to verify, while the distribution over $\rightarrow$ requires a proof.

Proposition 3. $[\varphi](\psi \wedge \chi) \equiv[\varphi] \psi \wedge[\varphi] \chi$

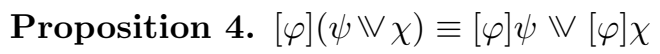

Proposition 5. $[\varphi](\psi \rightarrow \chi) \equiv[\varphi] \psi \rightarrow[\varphi] \chi$

Proof. We have the following, where the crucial passage from the second to the third line is justified by the set-theoretic fact that the subsets of $s \cap|\varphi|_{M}$ are all and only the sets of the form $t \cap|\varphi|_{M}$ for some $t \subseteq s$.

$$
\begin{aligned}
M, s \models[\varphi](\psi \rightarrow \chi) & \Longleftrightarrow M^{\varphi}, s \cap|\varphi|_{M}=\psi \rightarrow \chi \\
& \Longleftrightarrow \forall t \subseteq s \cap|\varphi|_{M}, \text { if } M^{\varphi}, t \models \psi \text { then } M^{\varphi}, t=\chi \\
& \Longleftrightarrow \forall t \subseteq s, \text { if } M^{\varphi}, t \cap|\varphi|_{M}=\psi \text { then } M^{\varphi}, t \cap|\varphi|_{M} \models \chi \\
& \Longleftrightarrow \forall t \subseteq s, \text { if } M, t \models[\varphi] \psi \text { then } M, t \models[\varphi] \chi \\
& \Longleftrightarrow M, s \models[\varphi] \psi \rightarrow[\varphi] \chi
\end{aligned}
$$

A dynamic modality $[\varphi]$ over a $K$ modality behaves as in PAL: it can be brought within the scope of $K$, provided that we condition the resulting formula on $\varphi$.

Proposition 6. $[\varphi] K_{a} \psi \equiv \varphi \rightarrow K_{a}[\varphi] \psi$

Proof. Notice that both $\varphi \rightarrow K_{a}[\varphi] \psi$ and $[\varphi] K_{a} \psi$ are declaratives, and thus truth-conditional. Hence, to establish the equivalence we just have to show that 
they have identical truth-conditions. Making use of Fact 2, we have:

$$
\begin{aligned}
M, w \models[\varphi] K_{a} \psi & \Longleftrightarrow M, w \models \varphi \text { implies } M^{\varphi}, w=K_{a} \psi \\
& \Longleftrightarrow M, w \models \varphi \text { implies } M^{\varphi}, \sigma_{a}^{\varphi}(w) \models \psi \\
& \Longleftrightarrow M, w \models \varphi \text { implies } M^{\varphi}, \sigma_{a}(w) \cap|\varphi|_{M} \models \psi \\
& \Longleftrightarrow M, w \models \varphi \text { implies } M, \sigma_{a}(w) \models[\varphi] \psi \\
& \Longleftrightarrow M, w \models \varphi \text { implies } M, w \models K_{a}[\varphi] \psi \\
& \Longleftrightarrow M, w \models \varphi \rightarrow K_{a}[\varphi] \psi
\end{aligned}
$$

The logical law that allows us to push a dynamic modality through an $E$ modality is only slightly more complex than the one for the $K$ modality. However, the proof of this law is rather complex, and requires some preliminary lemmata. For this reason, the proof is given in Appendix A.2.

Proposition 7. $[\varphi] E_{a} \psi \equiv \varphi \rightarrow E_{a}(\varphi \rightarrow[\varphi] \psi)$

One may wonder whether this proposition could not be simplified. After all, in PAL, it holds generally that $\varphi \rightarrow[\varphi] \psi \equiv[\varphi] \psi$, because $[\varphi] \psi$ is always true in a world where $\varphi$ is false. If this law held in IDEL as well, we could make the equivalence for the $E_{a}$ modality simpler, and completely analogous to the equivalence we stated for $K_{a}$. This is indeed the case if $\varphi$ is truth-conditional.

Proposition 8. If $\varphi \in \mathcal{L}^{I D E L}$ is truth-conditional, $\varphi \rightarrow[\varphi] \psi \equiv[\varphi] \psi$

The proof is given in Appendix A.3. However, this does not extend to the case in which $\varphi$ is a question. For a counterexample, one can check by inspecting the support clauses that $? p \rightarrow[? p] ? p \equiv \top$, while $[? p] ? p \equiv ? p$. This shows that, in general, $\varphi \rightarrow[\varphi] \psi \not \equiv[\varphi] \psi$ in IDEL; thus, Proposition 7 cannot be simplified.

Equipped with propositions 2-7, we are now ready to prove that any IDELformula is equivalent to some formula in the static language of IEL.

Theorem 2. For any $\varphi \in \mathcal{L}^{I D E L}, \varphi \equiv \varphi^{*}$ for some $\varphi^{*} \in \mathcal{L}^{I E L}$.

Proof. By induction on the structure of $\varphi$. The only step which is not immediate is the one for $\varphi=[\psi] \chi$. By induction hypothesis, we have two formulas $\psi^{*}, \chi^{*} \in$ $\mathcal{L}^{\mathrm{IEL}}$ which are equivalent to $\psi$ and $\chi$ respectively. Thus, $\varphi \equiv\left[\psi^{*}\right] \chi^{*}$. Now we just have to show that $\left[\psi^{*}\right] \chi^{*} \equiv \varphi^{*}$ for some $\varphi^{*} \in \mathcal{L}^{\mathrm{IEL}}$. For this, we proceed by induction on the complexity of $\chi^{*}$. Since $\chi^{*} \in \mathcal{L}^{\mathrm{IEL}}$, we only have to consider the base cases (atoms and $\perp$ ) and the inductive steps for the connectives and for $K_{a}$ and $E_{a}$. Each of these cases corresponds precisely to one of the equivalences that we just established. As an illustration, we give the inductive step for $E$.

- Suppose $\chi^{*}=E_{a} \xi$. By Proposition 7 we have $\varphi \equiv\left[\psi^{*}\right] \chi^{*}=\left[\psi^{*}\right] E_{a} \xi \equiv \psi^{*} \rightarrow$ $E_{a}\left(\psi^{*} \rightarrow\left[\psi^{*}\right] \xi\right)$. Now, $\xi$ is less complex than $\chi^{*}$, so by induction hypothesis there is a $\xi^{*} \in \mathcal{L}^{\mathrm{IEL}}$ such that $\left[\psi^{*}\right] \xi \equiv \xi^{*}$. Hence, $\varphi \equiv \psi^{*} \rightarrow E_{a}\left(\psi^{*} \rightarrow \xi^{*}\right)$. Since both $\psi^{*}$ and $\xi^{*}$ are in $\mathcal{L}^{\mathrm{IEL}}$, so is the formula $\psi^{*} \rightarrow E_{a}\left(\psi^{*} \rightarrow \xi^{*}\right)$, which we can thus take to be the desired $\varphi^{*}$. 


\begin{tabular}{|c|c|c|c|}
\hline !Atom & $! \perp$ & $! \wedge$ & $! \rightarrow$ \\
\hline$[\varphi] p$ & {$[\varphi] \perp$} & {$[\varphi](\psi \wedge \chi)$} & {$[\varphi](\psi \rightarrow \chi)$} \\
\hline$\overline{\varphi \rightarrow p}$ & $\overline{7 \overline{ }}$ & $\overline{[\varphi \varphi] \psi \wedge[\varphi] \chi}$ & $\overline{\overline{[\varphi] \psi} \rightarrow[\varphi] \chi}$ \\
\hline$! \mathbb{V}$ & $! K$ & $! E$ & $\mathrm{RE}$ \\
\hline 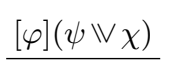 & {$[\varphi] K_{a} \psi$} & {$[\varphi] E_{a} \psi$} & $\varphi \leftrightarrow \psi$ \\
\hline$\overline{\overline{[\varphi] \psi \backslash \mathbb{V}[\varphi] \chi}}$ & $\overline{\overline{\varphi \rightarrow K_{a}[\varphi] \psi}}$ & $\overline{\overline{\varphi \rightarrow E_{a}(\varphi \rightarrow[\varphi] \psi)}}$ & $\overline{\chi[\varphi / p] \leftrightarrow \chi[\psi / p]}$ \\
\hline
\end{tabular}

Fig. 3. Inference rules for dynamic modalities. All but the last rule are bi-directional.

\section{Axiomatizing IDEL}

We can use the reduction of IDEL to IEL to provide a complete axiomatization for IDEL. All we need to do is to enrich a complete system for IEL, such as the natural deduction system given in [4], with inference rules which allow us to perform the reduction. The easiest way to achieve this is to turn the equivalences given by Proposition 2-7 into inference rules, and to equip our system with a rule of replacement of equivalents. These rules are shown in Fig. 3. We will denote the relation of derivability in this system by $\vdash_{\mathrm{IDEL}}{ }^{\mathrm{RE}}$, and the relation of inter-

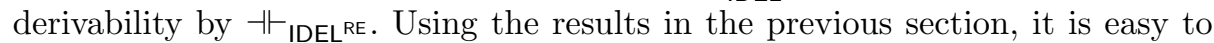
show that this system is sound for IDEL. The next proposition states that this system can prove any formula $\varphi \in \mathcal{L}^{\mathrm{IDEL}}$ to be equivalent to some $\varphi^{*} \in \mathcal{L}^{\mathrm{IEL}}$.

Proposition 9. For any $\varphi \in \mathcal{L}^{I D E L}, \varphi \vdash_{I D E L^{R E}} \varphi^{*}$ for some $\varphi^{*} \in \mathcal{L}^{I E L}$.

Proof. We proceed as in the proof of Theorem 2, replacing $\equiv$ by $\vdash_{\text {IDEL RE }}$.

The completeness of our system follows immediately from this and from the fact that our system includes a complete system for IEL.

Theorem 3. For any $\Phi \cup\{\psi\} \subseteq \mathcal{L}^{I D E L}, \Phi \models \psi \Longleftrightarrow \Phi \vdash_{I D E L^{R E}} \psi$.

The system described in Figure 3 gives a simple axiomatization for IDEL, similar to the axiomatization of PAL given in [16]. As in the case of PAL, there are some interesting alternatives that we can use instead of replacement of equivalents to ensure that the system can perform the reduction to IEL. One option is to notice that, like in PAL, two dynamic modalities can always be merged into a single, complex one. This gives the following composition law for dynamic modalities. The non-trivial proof of this result is given in Appendix A.4.

Proposition 10 (Composition law). $[\varphi][\psi] \chi \equiv[\varphi \wedge[\varphi] \psi] \chi$

We can turn this equivalence into a bidirectional inference rule, denoted !Comp. Now consider the proof system $\vdash_{\text {IDEL }}$ !Comp which is just like $\vdash_{\text {IDEL }}{ }^{R E}$, except that the rule RE is substituted by !Comp. The next theorem states that this system is sound and complete for IDEL. The proof is given in Appendix A.5. 
Theorem 4. For any $\Phi \cup\{\psi\} \subseteq \mathcal{L}^{I D E L}, \Phi \models \psi \Longleftrightarrow \Phi \vdash_{\text {IDEL' }}^{\text {Comp }} \psi$.

This is analogous to the axiomatization of PAL given in Theorem 7.26 of [11]. Finally, there is yet another alternative that we can use, instead of the rules RE and !Comp. This builds on the next proposition, which ensures that dynamic modalities are monotonic. The straightforward proof is omitted.

Proposition 11 (Monotonicity). If $\psi \models \chi$, then $[\varphi] \psi \models[\varphi] \chi$.

We can turn this logical property into an inference rule, that we will call !Mon:

!Mon: given $[\varphi] \psi$ and given a proof of $\chi$ from $\psi$, infer $[\varphi] \chi$.

Let us denote by $\vdash_{\text {IDEL'Mon }}$ the system which is just like $\vdash_{\text {IDEL }}$, except that the rule of replacement of equivalents is substituted by !Mon. The next theorem, proved in Appendix A.5, says that this system, too, is complete for IDEL.

Theorem 5. For any $\Phi \cup\{\psi\} \subseteq \mathcal{L}^{I D E L}, \Phi \models \psi \Longleftrightarrow \Phi \vdash_{I D E L L^{\prime M o n}} \psi$.

This is similar to the axiomatization of PAL given by Corollary 12 of [17]. We have thus established three different complete axiomatizations for IDEL, each of which is the analogue of one known axiomatization of PAL.

\section{Conclusion and outlook}

In this paper we have investigated the logic of public utterance in IDEL, and found that it has much in common with standard public announcement logic. Just like in PAL, dynamic modalities can be recursively eliminated, turning each formula into an equivalent static formula. We have exploited this fact to establish three axiomatizations of IDEL, corresponding to existing axiomatizations of PAL. The first axiomatization contains, in addition to a complete set of rules for inquisitive epistemic logic, the reduction equivalences and the rule of replacement of equivalents. In the other two axiomatizations, replacement of equivalents is not used; instead, we use one of two other features which IDEL shares with PAL:

(i) the fact that two dynamic modalities can be composed into a single one and

(ii) the fact that dynamic modalities are monotonic.

These results are exciting, as they show that the standard logical analysis of public announcements, when lifted to an inquisitive semantics framework, can be generalized smoothly to an analysis of public utterance that deals not only with the effect of making statements, but also with the effect of asking questions. This provides us with a well-behaved logic to reason about how a multi-agent situation evolves not only as a result of new incoming information, but also as a result of new issues being raised. Besides being interesting in its own right, this justifies a more general hope that the wealth of results developed in the field of dynamic epistemic logics can be generalized to cover questions. The outcome of this would be a more comprehensive analysis of communication as a process in which agents interact by requesting and providing information. 


\section{References}

1. Alexandru Baltag, Lawrence S. Moss, and Slawomir Solecki. The logic of public announcements, common knowledge, and private suspicions. In Proceedings of the 7th Conference on Theoretical Aspects of Rationality and Knowledge, pages 43-56. Morgan Kaufmann Publishers, 1998.

2. Johan van Benthem. Logical dynamics of information and interaction. Cambridge University Press, 2011.

3. Ivano Ciardelli. Inquisitive semantics and intermediate logics. Master Thesis, University of Amsterdam, 2009.

4. Ivano Ciardelli. Modalities in the realm of questions: axiomatizing inquisitive epistemic logic. In Rajeev Goré, Barteld Kooi, and Agi Kurucz, editors, Advances in Modal Logic, pages 94-113, London, 2014. College Publications.

5. Ivano Ciardelli. Questions in logic. PhD thesis, ILLC, University of Amsterdam, 2016.

6. Ivano Ciardelli, Jeroen Groenendijk, and Floris Roelofsen. Inquisitive semantics: a new notion of meaning. Language and Linguistics Compass, 7(9):459-476, 2013.

7. Ivano Ciardelli, Jeroen Groenendijk, and Floris Roelofsen. On the semantics and logic of declaratives and interrogatives. Synthese, 192(6):1689-1728, 2015.

8. Ivano Ciardelli and Floris Roelofsen. Inquisitive logic. Journal of Philosophical Logic, 40(1):55-94, 2011.

9. Ivano Ciardelli and Floris Roelofsen. Inquisitive dynamic epistemic logic. Synthese, 192(6):1643-1687, 2015.

10. Hans van Ditmarsch. Knowledge Games. PhD thesis, University of Groningen, 2000 .

11. Hans van Ditmarsch, Wiebe van der Hoek, and Barteld Kooi. Dynamic Epistemic Logic. Springer, 2007.

12. Jelle Gerbrandy and Willem Groeneveld. Reasoning about information change. Journal of logic, language and information, 6(2):147-169, 1997.

13. Jeroen Groenendijk. Erotetic languages and the inquisitive hierarchy. In a Festschrift for Martin Stokhof, 2011.

14. Yacin Hamami. The interrogative model of inquiry meets dynamic epistemic logics. Synthese, 192(6):1609-1642, 2015.

15. Jaakko Hintikka. Inquiry as inquiry: A logic of scientific discovery. Kluwer Academic Publishers, 1999.

16. Jan Plaza. Logics of public communications. In M.L. Emrich, M.S. Pfeifer, M. Hadzikadic, and Z.W. Ras, editors, Proceedings of the Fourth International Symposium on Methodologies for Intelligent Systems, pages 201-216. Oak Ridge National Laboratory, 1989.

17. Yanjing Wang and Qinxiang Cao. On axiomatizations of public announcement logic. Synthese, 190(1):103-134, 2013. 


\section{A.1 Proof Theorem 1 (Normal Form)}

The proof is by induction on $\varphi$. For the base case, the claim is trivial. The inductive steps for conjunction, inquisitive disjunction, and implication are proved in the same way as for propositional inquisitive logic (see [7]). We are left with the inductive step for the dynamic modality. Let $\mathcal{R}(\psi)=\left\{\alpha_{1}, \ldots, \alpha_{n}\right\}$, and consider the formula $[\varphi] \psi$. Using the induction hypothesis on $\psi$, we have:

$$
\begin{aligned}
M, s \models[\varphi] \psi & \Longleftrightarrow M^{\varphi}, s \cap|\varphi|_{M} \models \psi \\
& \Longleftrightarrow M^{\varphi}, s \cap|\varphi|_{M}=\alpha_{1} \mathbb{V} \ldots \mathbb{V} \alpha_{n} \\
& \Longleftrightarrow M^{\varphi}, s \cap|\varphi|_{M}=\alpha_{i} \text { for some } 1 \leq i \leq n \\
& \Longleftrightarrow M, s=[\varphi] \alpha_{i} \text { for some } 1 \leq i \leq n \\
& \Longleftrightarrow M, s=[\varphi] \alpha_{1} \mathbb{V} \ldots \mathbb{V}[\varphi] \alpha_{n}
\end{aligned}
$$

Since $\mathcal{R}([\varphi] \psi)=\left\{[\varphi] \alpha_{1}, \ldots,[\varphi] \alpha_{n}\right\}$, this is what we needed to show.

\section{A.2 Proof of Proposition 7 (Reduction law for the $E$ modality)}

To prove Proposition 7, we first need some lemmata. As a first step, we show that the updated inquisitive state $\Sigma_{a}^{\varphi}(w)$ of an agent is obtained as the union, for $\alpha$ a resolution of $\varphi$, of the restriction of $\Sigma_{a}(w)$ to the $\alpha$-worlds.

Lemma 1. Let $\varphi \in \mathcal{L}^{I D E L}$ and let $\mathcal{R}(\varphi)=\left\{\alpha_{1}, \ldots, \alpha_{n}\right\}$. Given any $M$ and $w$ :

$$
\Sigma_{a}^{\varphi}(w)=\left.\left.\Sigma_{a}(w)\right|_{\left|\alpha_{1}\right|_{M}} \cup \ldots \cup \Sigma_{a}(w)\right|_{\left|\alpha_{n}\right|_{M}}
$$

Proof. Theorem 1 ensures that $\varphi \equiv \alpha_{1} \mathbb{V} \ldots \mathbb{V} \alpha_{n}$. Given the support clause for $\mathbb{V}$, this implies $[\varphi]_{M}=\left[\alpha_{1}\right]_{M} \cup \cdots \cup\left[\alpha_{n}\right]_{M}$. Thus, we have:

$$
\begin{aligned}
\Sigma_{a}^{\varphi}(w) & =\Sigma_{a}(w) \cap[\varphi]_{M}=\Sigma_{a}(w) \cap\left(\left[\alpha_{1}\right]_{M} \cup \cdots \cup\left[\alpha_{n}\right]_{M}\right) \\
& =\bigcup_{1 \leq i \leq n}\left(\Sigma_{a}(w) \cap\left[\alpha_{i}\right]_{M}\right)=\Sigma_{a}^{\alpha_{1}}(w) \cup \cdots \cup \Sigma_{a}^{\alpha_{n}}(w)
\end{aligned}
$$

Since resolutions are declaratives and thus truth-conditional, we have by Fact 3 that $\Sigma_{a}^{\alpha_{i}}(w)=\Sigma_{a}(w)\left\lceil\left|\alpha_{i}\right|_{M}\right.$. Thus, we have $\Sigma_{a}^{\varphi}(w)=\bigcup_{1 \leq i \leq n} \Sigma_{a}(w)\left\lceil\left|\alpha_{i}\right|_{M}\right.$.

We will also make use of the next lemma, stating that whenever the antecedent of an implication is a truth-conditional formula $\alpha$, the clause for implication can be simplified: $\alpha \rightarrow \psi$ is supported at $s$ iff $\psi$ is supported at the state $s \cap|\alpha|_{M}$ which results from extending $s$ with the information that $\alpha$ is true.

Lemma 2. Let $\alpha \in \mathcal{L}^{I D E L}$ be truth-conditional. Then for any $M$, s, and $\psi$ :

$$
M,\left.s|=\alpha \rightarrow \psi \Longleftrightarrow M, s \cap| \alpha\right|_{M} \models \psi
$$

Proof. If $\alpha$ is truth-conditional, then the states in $M$ which support $\alpha$ are all and only the subsets of $|\alpha|_{M}$. Now suppose $M, s \models \alpha \rightarrow \psi$. Since $s \cap|\alpha|_{M} \subseteq|\alpha|_{M}$, 
we have $M, s \cap|\alpha|_{M}=\alpha$. Since $s \cap|\alpha|_{M} \subseteq s$ and $M, s=\alpha \rightarrow \psi$, by the clause for $\rightarrow$ we have $M, s \cap|\alpha|_{M}=\psi$, which shows the left-to-right direction.

Conversely, suppose $M, s \cap|\alpha|_{M}=\psi$. Let $t \subseteq s$ with $M, t \models \alpha$ : this means that $t \subseteq|\alpha|_{M}$; since $t \subseteq s$, we have $t \subseteq s \cap|\alpha|_{M}$. Since $M, s \cap|\alpha|_{M} \mid=\psi$, it follows by persistency that $M, t \models \psi$. This shows that $M, s \models \alpha \rightarrow \psi$.

Finally, we will make use of the following equivalence, which can be established simply by spelling out the support conditions for the two formulas.

Lemma 3. For any $\varphi, \psi, \chi \in \mathcal{L}^{I D E L},(\varphi \vee \psi) \rightarrow \chi \equiv(\varphi \rightarrow \chi) \wedge(\psi \rightarrow \chi)$

Equipped with these tools, we are now ready to prove the reduction law given by Proposition 7 for the entertain modality.

Proof of Proposition 7. Let $\mathcal{R}(\varphi)=\left\{\alpha_{1}, \ldots, \alpha_{n}\right\}$. First, notice that, by Lemma 1, the information states $s \in \Sigma_{a}^{\varphi}(w)$ are all and only the information states of the form $s=t \cap\left|\alpha_{i}\right|_{M}$ for some $t \in \Sigma_{a}(w)$ and some $\alpha_{i} \in \mathcal{R}(\varphi)$. Since $\alpha_{i} \in \mathcal{R}(\varphi)$, it follows from Theorem 1 that $\left|\alpha_{i}\right|_{M} \subseteq|\varphi|_{M}$, whence $t \cap\left|\alpha_{i}\right|_{M}=t \cap\left|\alpha_{i}\right|_{M} \cap|\varphi|_{M}$.

Now suppose that $M, w \models \varphi$, so that $w$ survives in the updated model $M^{\varphi}$. Making use of these facts, of Theorem 1 and of lemmata 2 and 3, we have:

$$
\begin{aligned}
M^{\varphi}, w=E_{a} \psi & \Longleftrightarrow \forall s \in \Sigma_{a}^{\varphi}(w), M^{\varphi}, s \models \psi \\
& \Longleftrightarrow \forall t \in \Sigma_{a}(w), \forall \alpha_{i} \in \mathcal{R}(\varphi), M^{\varphi}, t \cap\left|\alpha_{i}\right|_{M} \models \psi \\
& \Longleftrightarrow \forall t \in \Sigma_{a}(w), \forall \alpha_{i} \in \mathcal{R}(\varphi), M^{\varphi}, t \cap\left|\alpha_{i}\right|_{M} \cap|\varphi|_{M} \models \psi \\
& \Longleftrightarrow \forall t \in \Sigma_{a}(w), \forall \alpha_{i} \in \mathcal{R}(\varphi), M, t \cap\left|\alpha_{i}\right|_{M} \models[\varphi] \psi \\
& \Longleftrightarrow \forall t \in \Sigma_{a}(w), \forall \alpha_{i} \in \mathcal{R}(\varphi), M, t \models \alpha_{i} \rightarrow[\varphi] \psi \\
& \Longleftrightarrow \forall t \in \Sigma_{a}(w), M, t \models\left(\alpha_{1} \rightarrow[\varphi] \psi\right) \wedge \cdots \wedge\left(\alpha_{n} \rightarrow[\varphi] \psi\right) \\
& \Longleftrightarrow \forall t \in \Sigma_{a}(w), M, t \models\left(\alpha_{1} \mathbb{\vee} \ldots \mathbb{V} \alpha_{n}\right) \rightarrow[\varphi] \psi \\
& \Longleftrightarrow \forall t \in \Sigma_{a}(w), M, t \models \varphi \rightarrow[\varphi] \psi \\
& \Longleftrightarrow M, w \models E_{a}(\varphi \rightarrow[\varphi] \psi)
\end{aligned}
$$

Finally, using this equivalence we get, for any model $M$ and world $w$ :

$$
\begin{aligned}
M, w \models[\varphi] E_{a} \psi & \Longleftrightarrow M, w=\varphi \text { implies } M^{\varphi}, w \models E_{a} \psi \\
& \Longleftrightarrow M, w=\varphi \text { implies } M, w \models E_{a}(\varphi \rightarrow[\varphi] \psi) \\
& \Longleftrightarrow M, w=\varphi \rightarrow E_{a}(\varphi \rightarrow[\varphi] \psi)
\end{aligned}
$$

We have thus shown that the formulas $[\varphi] E_{a} \psi$ and $\varphi \rightarrow E_{a}(\varphi \rightarrow[\varphi] \psi)$ have the same truth-conditions. But both formulas are declaratives, and thus truthconditional by Proposition 1. It follows that these formulas are equivalent.

\section{A.3 Proof of Proposition 8 (Antecedent simplification)}

Suppose $\varphi$ is truth-conditional, and consider any model $M$ and state $s$. Making use of Lemma 2 on implications with a truth-conditional antecedent, we have:

$$
\begin{aligned}
M, s=\varphi \rightarrow[\varphi] \psi & \Longleftrightarrow M, s \cap|\varphi|_{M}=[\varphi] \psi \\
& \Longleftrightarrow M^{\varphi}, s \cap|\varphi|_{M} \cap|\varphi|_{M} \models \psi \\
& \Longleftrightarrow M^{\varphi}, s \cap|\varphi|_{M} \models \psi \Longleftrightarrow M, s \models[\varphi] \psi
\end{aligned}
$$




\section{A.4 Proof of Proposition 10 (Composition of dynamic modalities)}

To show the validity of the composition law for dynamic modalities, we start by proving that a sequence of two updates of a model can always be simulated by a single update, just as in PAL.

Lemma 4 (Update composition).

For any formulas $\varphi, \psi \in \mathcal{L}^{I D E L}$ and for any model $M:\left(M^{\varphi}\right)^{\psi}=M^{\varphi \wedge[\varphi] \psi}$

Proof In order to show that $\left(M^{\varphi}\right)^{\psi}=M^{\varphi \wedge[\varphi] \psi}$, let us start out by showing that the sets of worlds $\left(W^{\varphi}\right)^{\psi}$ and $W^{\varphi \wedge[\varphi] \psi}$ are the same. We have

$$
W^{\varphi \wedge[\varphi] \psi}=W \cap|\varphi \wedge[\varphi] \psi|_{M}=W \cap|\varphi|_{M} \cap|[\varphi] \psi|_{M}
$$

Now, if we take a world $w \in W$ such that $M, w \models \varphi$, then $M, w \models[\varphi] \psi \Longleftrightarrow$ $M^{\varphi}, w \mid=\psi$. This means that $|\varphi|_{M} \cap|[\varphi] \psi|_{M}=|\varphi|_{M} \cap|\psi|_{M \varphi}$. So, we have:

$$
W \cap|\varphi|_{M} \cap|[\varphi] \psi|_{M}=W \cap|\varphi|_{M} \cap|\psi|_{M^{\varphi}}=W^{\varphi} \cap|\psi|_{M^{\varphi}}=\left(W^{\varphi}\right)^{\psi}
$$

This shows that the models $\left(M^{\varphi}\right)^{\psi}$ and $M^{\varphi \wedge[\varphi] \psi}$ share the same universe of possible worlds. Since the truth-value of a propositional atom at a world is not affected by updates, the two also have the same valuation function. Now let us consider the state map for an agent $a$ at a world $w$. We have:

$$
\Sigma_{a}^{\varphi \wedge[\varphi] \psi}(w)=\Sigma_{a}(w) \cap[\varphi \wedge[\varphi] \psi]_{M}=\Sigma_{a}(w) \cap[\varphi]_{M} \cap[[\varphi] \psi]_{M}
$$

Now, consider a state $s \in[\varphi]_{M}$. We have $s \subseteq \bigcup[\varphi]_{M}=|\varphi|_{M}$, so $s \cap|\varphi|_{M}=s$. So, for such an $s$ we have $M, s=[\varphi] \psi \Longleftrightarrow M^{\varphi}, s \cap|\varphi|_{M}=\psi \Longleftrightarrow M^{\varphi}, s \models \psi$. This shows that $[\varphi]_{M} \cap[[\varphi] \psi]_{M}=[\varphi]_{M} \cap[\psi]_{M \varphi}$. Using this fact, we obtain:

$\Sigma_{a}(w) \cap[\varphi]_{M} \cap[[\varphi] \psi]_{M}=\Sigma_{a}(w) \cap[\varphi]_{M} \cap[\psi]_{M^{\varphi}}=\Sigma_{a}^{\varphi}(w) \cap[\psi]_{M \varphi}=\left(\Sigma_{a}^{\varphi}\right)^{\psi}(w)$

Since this holds for any world $w$, the models $\left(M^{\varphi}\right)^{\psi}$ and $M^{\varphi \wedge[\varphi] \psi}$ also have the same state map for each agent, which completes the proof.

We can now use our findings to prove the composition law of Proposition 10.

Proof of Proposition 10. Take any model $M$ and any state $s$. In the previous proof, we have established that $\left(M^{\varphi}\right)^{\psi}=M^{\varphi \wedge[\varphi] \psi}$ and also that $|\varphi \wedge[\varphi] \psi|_{M}=$ $|\varphi|_{M} \cap|\psi|_{M \varphi}$. Making use of these facts, we have:

$$
\begin{aligned}
M, s \models[\varphi][\psi] \chi & \Longleftrightarrow M^{\varphi}, s \cap|\varphi|_{M}=[\psi] \chi \\
& \left.\Longleftrightarrow M^{\varphi}\right)^{\psi}, s \cap|\varphi|_{M} \cap|\psi|_{M^{\varphi}} \models \chi \\
& \Longleftrightarrow M^{\varphi \wedge[\varphi] \psi}, s \cap|\varphi \wedge[\varphi] \psi|_{M} \models \chi \\
& \Longleftrightarrow M, s=[\varphi \wedge[\varphi] \psi] \chi
\end{aligned}
$$

\section{A.5 Proof of completeness via !Comp and via !Mon}

Proof of Theorem 4 The proof is analogous to the one given in $\S 7.4$ of [11] for PAL. In the interest of conciseness, we omit the straightforward parts of the proof. First, we define a complexity measure $c: \mathcal{L}^{\mathrm{IDEL}} \rightarrow \mathbb{N}$ as follows: 
$-c(p)=c(\perp)=1$

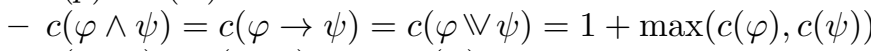

- $c\left(K_{a} \varphi\right)=c\left(E_{a} \varphi\right)=1+c(\varphi)$

$-c([\varphi] \psi)=(4+c(\varphi)) \cdot c(\psi)$

Next, we make sure that this measure satisfies the following conditions:

$-c(\varphi)>c(\psi)$ if $\psi$ is a proper sub-formula of $\varphi$

$-c([\varphi] p)>c(\varphi \rightarrow p)$ and $c([\varphi] \perp)>c(\neg \varphi)$

- $c([\varphi](\psi \circ \chi))>c([\varphi] \psi \circ[\varphi] \chi)$ for $\circ \in\{\wedge, \rightarrow, \mathbb{V}\}$

$-c\left([\varphi] K_{a} \psi\right)>c\left(\varphi \rightarrow K_{a}[\varphi] \psi\right)$

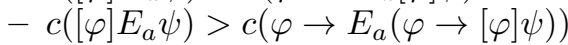

$-c([\varphi][\psi] \chi)>c([\varphi \wedge[\varphi] \psi] \chi)$

By recursion on this notion of complexity, we define a map $(\cdot)^{*}: \mathcal{L}^{\mathrm{IDEL}} \rightarrow \mathcal{L}^{\mathrm{IEL}}$ :

$$
\begin{array}{ll}
p^{*}=p, \quad \perp^{*}=\perp & ([\varphi](\psi \wedge \chi))^{*}=([\varphi] \psi \wedge[\varphi] \chi)^{*} \\
(\varphi \wedge \psi)^{*}=\varphi^{*} \wedge \psi^{*} & ([\varphi](\psi \rightarrow \chi))^{*}=([\varphi] \psi \rightarrow[\varphi] \chi)^{*} \\
(\varphi \rightarrow \psi)^{*}=\varphi^{*} \rightarrow \psi^{*} & ([\varphi](\psi \vee \chi))^{*}=([\varphi] \psi \mathbb{\varphi}[\varphi] \chi)^{*} \\
(\varphi \backslash \psi)^{*}=\varphi^{*} \backslash \psi^{*} & \left([\varphi] K_{a} \psi\right)^{*}=\left(\varphi \rightarrow K_{a}[\varphi] \psi\right)^{*} \\
([\varphi] p)^{*}=(\varphi \rightarrow p)^{*} & \left([\varphi] E_{a} \psi\right)^{*}=\left(\varphi \rightarrow E_{a}(\varphi \rightarrow[\varphi] \psi)\right)^{*} \\
([\varphi] \perp)^{*}=(\neg \varphi)^{*} & ([\varphi][\psi] \chi)^{*}=([\varphi \wedge[\varphi] \psi] \chi)^{*}
\end{array}
$$

By induction on the complexity of $\varphi$, we can then easily prove $\varphi \nvdash_{\text {IDEL IComp }} \varphi^{*}$, using the reduction rules and the rule !Comp. Completeness then follows from the fact that $\vdash_{\text {IDEL!Comp }}$ includes a complete system for IEL.

Proof of Theorem 5. The proof is very similar to the proof of Theorem 4 (and similar to the proof of the analogous result for PAL in [17]). We modify the definition of $(\cdot)^{*}$ slightly by setting $([\varphi][\psi] \chi)^{*}=\left([\varphi]([\psi] \chi)^{*}\right)^{*}$.

By induction on the complexity of a formula (as defined above), we show that (i) $\varphi^{*}$ is well-defined; (ii) if $\varphi \notin \mathcal{L}^{\text {IEL }}$, then $c\left(\varphi^{*}\right)<c(\varphi)$; and (iii) $\varphi \dashv_{\text {IDEL 'Mon }} \varphi^{*}$. The only case which is not straightforward is the inductive step for a formula of the form $[\varphi][\psi] \chi$, which I will spell out in detail.

First, notice that since $[\psi] \chi$ is less complex than $[\varphi][\psi] \chi$, by induction hypothesis we have that $([\psi] \chi)^{*}$ is well-defined and less complex than $[\psi] \chi$. It follows that $[\varphi]([\psi] \chi)^{*}$ is less complex than $[\varphi][\psi] \chi$. So, the induction hypothesis implies that $\left([\varphi]([\psi] \chi)^{*}\right)^{*}$ is well-defined, i.e., that $([\varphi][\psi] \chi)^{*}$ is well-defined.

Second, as both $[\psi] \chi$ and $[\varphi]([\psi] \chi)^{*}$ are less complex than $[\varphi][\psi] \chi$, using the induction hypothesis we have $c\left(([\varphi][\psi] \chi)^{*}\right)=c\left(\left([\varphi]([\psi] \chi)^{*}\right)^{*}\right)<c\left([\varphi]([\psi] \chi)^{*}\right)<$ $c([\varphi][\psi] \chi)$. So, $c\left(([\varphi][\psi] \chi)^{*}\right)<c([\varphi][\psi] \chi)$.

Finally, since $[\psi] \chi$ is less complex than $[\varphi][\psi] \chi$, the induction hypothesis gives $[\psi] \chi-\nVdash_{\text {IDEL'Mon }}([\psi] \chi)^{*}$. By two applications of the rule !Mon we get $[\varphi][\psi] \chi \dashv \nVdash_{\text {IDEL'Mon }}[\varphi]([\psi] \chi)^{*}$. Now, since $[\varphi]([\psi] \chi)^{*}$ is less complex than $[\varphi][\psi] \chi$, the induction hypothesis applies, and gives $[\varphi]([\psi] \chi)^{*} \dashv \vdash_{\text {IDEL!Mon }}\left([\varphi]([\psi] \chi)^{*}\right)^{*}$. Putting things together, we have obtained $[\varphi][\psi] \chi-\nVdash_{\text {IDEL!Mon }}\left([\varphi]([\psi] \chi)^{*}\right)^{*}$ which is what we need, since by definition $\left([\varphi]([\psi] \chi)^{*}\right)^{*}=([\varphi][\psi] \chi)^{*}$.

Thus, our system proves any $\varphi \in \mathcal{L}^{\text {IDEL }}$ to be equivalent to some $\varphi^{*} \in \mathcal{L}^{\mathrm{IEL}}$. Since our system is complete for IEL, the completeness result follows. 